Document downloaded from:

http://hdl.handle.net/10251/36442

This paper must be cited as:

Yuichi Nagata; Soler Fernández, D. (2012). A new genetic algorithm for the asymmetric traveling salesman problem. Expert Systems with Applications. 39(10):8947-8953. doi:10.1016/j.eswa.2012.02.029.

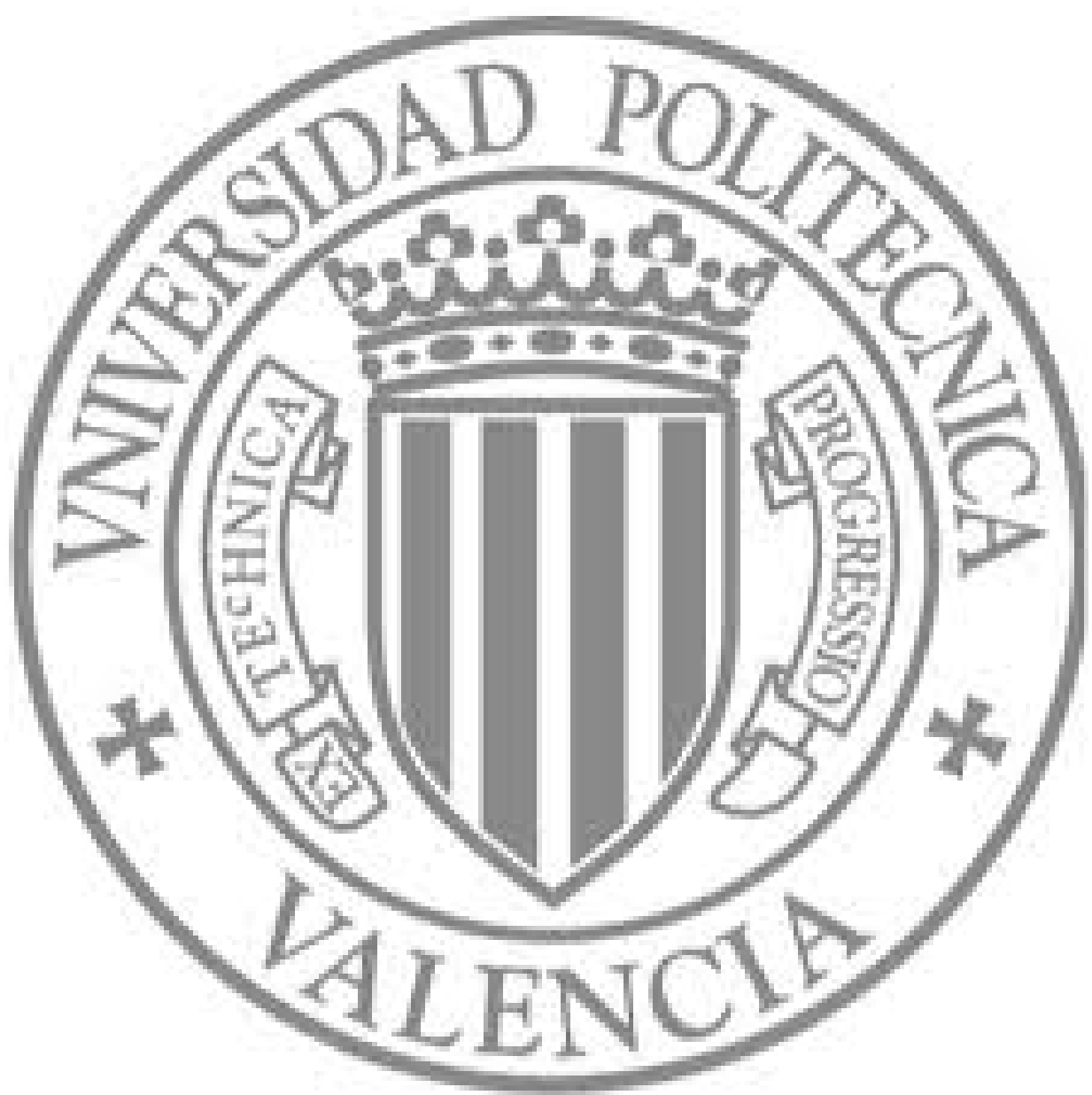

The final publication is available at

http://dx.doi.org/10.1016/j.eswa.2012.02.029

Copyright Elsevier 


\title{
A new genetic algorithm for the asymmetric traveling salesman problem
}

\author{
Yuichi Nagata ${ }^{a, *}$, David Soler ${ }^{b}$ \\ ${ }^{a}$ Tokyo Institute of Technology, Kanagawa, Japan \\ ${ }^{b}$ Institut Universitari de Matemàtica Pura i Aplicada, \\ Universitat Politècnica de València, València, Spain
}

\begin{abstract}
The asymmetric traveling salesman problem (ATSP) is one of the most important combinatorial optimization problems. It allows us to solve, either directly or through a transformation, many real-world problems. We present in this paper a new competitive genetic algorithm to solve this problem. This algorithm has been checked on a set of 153 benchmark instances with known optimal solution and it outperforms the results obtained with previous ATSP heuristic methods.
\end{abstract}

Keywords: Asymmetric traveling salesman problem, genetic algorithm, crossover operator, metaheuristics

\section{Introduction}

The asymmetric traveling salesman problem (ATSP) is one of the most important and well-known problems in combinatorial optimization, and it can be stated as follows:

${ }^{*}$ Correspondence: Y. Nagata, Interdisciplinary Graduate School of Science and Engineering, Tokyo Institute of Technology, 4259 Nagatsuta Midori-ku Yokohama, Kanagawa 226-8502, Japan. E-mail: nagata@fe.dis.titech.ac.jp. 
Given a complete directed graph $G=(V, A), V$ being the vertex set and $A$ being the arc set, with nonnegative costs associated with its arcs, find a minimum cost circuit in $G$ passing through each vertex exactly once.

The ATSP has important applications to real-world problems, especially in sequencing, in distribution, and in vehicle routing problems. As a result, dozens of papers and several surveys have been written about ATSP in the past few decades. To be brief, we only mention the classical survey by Fischetti et al. (2002), detailing different exact procedures for the ATSP; the most recent exact procedure by Corberan et al. (2005), capable of solving large-size ATSP instances; the latest competitive heuristic approach for solving the ATSP by Xing et al. (2008), whose article also serves as a good survey on metaheuristic approaches for solving the ATSP; the work by Oncan et al. (2009), in which different formulations for the ATSP are compared; and the very recent concise guide by Laporte (2010) and survey on local search algorithms for this problem by Rego et al. (2011).

In addition to its direct applications to real-world problems, a review of the literature shows that many single-vehicle routing problems, which actually cannot be modeled as an ATSP, can be solved through a polynomial transformation into an ATSP. The main advantage of this transformation is that efficient algorithms developed for the ATSP can be directly applied to these problems without any modification.

Among the problems that can be solved through a transformation into an ATSP are several classes of arc and/or node routing problems, defined on undirected, directed, or mixed graphs, some of which include turn penalties, forbidden turns, time-dependent costs, or delivery time windows. In this area, we cite the works by Laporte (1997), Clossey et al. (2001), Corberán et al. (2002), Blais and Laporte (2003), Soler et al. (2008), and Albiach et al. (2008). In most of these articles, the transformations they propose consist of two steps. They first transform their problem into another combinatorial optimization problem, namely the asymmetric generalized traveling salesman problem (AGTSP), which in turn can be transformed into an ATSP (see, e.g., Noon and Bean (1993) or Ben-Arieh et al. (2003)). The AGTSP is actually a generalization of the ATSP in which each customer has several alternative locations and only one of them has to be selected for service. It can be briefly defined as follows: 
Given a directed graph $G=(V, A)$ with nonnegative costs associated with its arcs, such that $V$ is partitioned into $k$ nonempty subsets $\left\{S_{i}\right\}_{i=1}^{k}$, find a minimum cost circuit passing through exactly one vertex of each subset $S_{i} \forall i \in\{1, \ldots, k\}$.

It is worth commenting here that the idea of solving a single-vehicle routing problem by transforming it into an ATSP through an AGTSP has been recently generalized to solve different hard multivehicle routing problems, as we can see through the papers by Soler et al. (2009), Baldacci et al. (2010), Bräysy et al. (2011), and Micó and Soler (2011).

All these facts have encouraged us to present a powerful new approximation algorithm to solve the ATSP, with the aim of improving results given by previous ATSP heuristics to help experts solve real-world problems (such as those cited above) or to measure the efficiency of actual or future proposed heuristics that directly address these problems. Our solution approach is based on genetic algorithms (GAs). The GA is a population-based approach for heuristic search in optimization problems, and it has been applied to a wide variety of optimization problems. In particular, many GAs or memetic algorithms (MAs) have been developed for the symmetric TSP (STSP) up to the present date (see, e.g., the very recent papers by Albayrak and Allahverdi (2011) and Chen and Chien (2011)). Here, the MA is also a population-based heuristic search that combines a GA with a local search to organize a more intensive local search. However, applications of the GA or MA to the ATSP are rather limited. Here we refer the reader to the three latest GAs and MAs proposed for the ATSP, all of which have shown good performance (see Choi et al. (2003), Buriol et al. (2004), and Xing et al. $(2008))$.

The main feature of our GA is the use of an edge assembly crossover (EAX) operator, which was originally proposed by Nagata and Kobayashi (1997) for both the STSP and the ATSP. The EAX generates offspring solutions by combining (undirected) edges or arcs from two parent solutions and adding relatively few short edges or arcs. The main difference between the EAX for the ATSP and the EAX for the STSP is that the first one combines parents' arcs (directed edges) without changing their orientation whereas the second one combines parents' (undirected) edges without respect to their orientation. Since a GA using an EAX was originally proposed, it has been significantly 
enhanced to improve the performance for solving the STSP; the capability of the EAX for generating good offspring solutions has been enhanced by Nagata (2004, 2006b) and the capability of the GA framework for maintaining the population diversity has been also enhanced by Nagata (2006a). Because of the growing importance of the ATSP, in this paper we investigate the performance of the GA using the EAX for the ATSP by applying similar enhancements to those developed for the STSP and introducing an additional enhancement to further improve the performance.

To check the efficiency of our GA, we have tested it on 153 benchmark ATSP instances, all of which have known optimal solutions. These instances correspond to two different sets: the 27 well-known instances from TSPLIB (http://comopt.ifi.uniheidelberg.de/software/TSPLIB95/) and 126 instances with up to 315 vertices from the work by Soler et al. (2008).

The rest of the paper is organized as follows. Section 2 presents the genetic algorithm for the ATSP, Section 3 shows the computational results obtained for the set of 153 instances, and Section 4 gives some conclusions about this work.

\section{The genetic algorithm}

In this section, we describe the GA framework and the EAX developed for the ATSP. In Section 2.1 we first present the EAX, which includes adapted enhancements developed for the STSP. The GA framework in which the EAX is integrated is then presented in Section 2.2. We present in Section 2.3 the local search procedure used for generating an initial population for the proposed GA.

\subsection{EAX for the ATSP}

The EAX for the ATSP consists of five steps as outlined below and illustrated in Figure 1. If more than two offspring solutions are generated, Steps 3-5 are repeated.

\section{[Basic algorithm]}

Step 1 Given parent solutions denoted as $p_{A}$ and $p_{B}$, let $G_{A B}$ be the directed graph defined as $G_{A B}=\left(V, E_{A} \cup E_{B} \backslash E_{A} \cap E_{B}\right)$, where $E_{A}$ and $E_{B}$ are defined as sets 
of arcs consisting of $p_{A}$ and $p_{B}$, respectively.

Step 2 Partition all edges of $G_{A B}$ into $A B$-cycles. An $A B$-cycle is defined as a cycle in $G_{A B}$ such that arcs from $E_{A}$ and $E_{B}$ are alternately linked in the opposite orientation. Note that partition of the arcs into $A B$-cycles is always possible and uniquely determined (which is easy to prove).

Step 3 Construct an E-set by selecting $A B$-cycles according to a given rule, where an E-set is defined as the union of AB-cycles.

Step 4 Generate an intermediate solution from $p_{A}$ by removing the arcs of $E_{A}$ and adding the $\operatorname{arcs}$ of $E_{B}$ in the $E$-set. As a result, an intermediate solution consists of one or more subtours as illustrated in Figure 1.

Step 5 Connect subtours into a tour to generate an offspring solution. More precisely, subtours are connected one by one, each time connecting a subtour consisting of the least number of arcs to one of the other subtours so that the sum of the cost of the arcs is minimized. Here, two subtours are connected by deleting one arc from each of the subtours and adding two arcs to connect them in such a way that all arcs in the resulting subtour have the same orientation.

In Step 3, an E-set of any combination of AB-cycles can be constructed, and the EAX can generate various intermediate solutions. One simple strategy, which was proposed in the original EAX (Nagata and Kobayashi, 1997), is to select a number of $A B$-cycles randomly, each with a probability of 0.5, to construct an E-set. An EAX with this selection strategy is called EAX-Rand and it typically forms an intermediate solution that contains arcs of $E_{A}$ and arcs of $E_{B}$ equally. An alternative strategy for selecting $A B$-cycles was proposed by Nagata (2004a) to enhance the EAX for the STSP. This strategy randomly selects a single $A B$-cycle without overlapping the previous selection. Therefore, an EAX with this strategy can generate at most the same number of offspring solutions as the number of $A B$-cycles generated. An EAX with this selection strategy is called EAX-1AB and typically forms an intermediate solution similar to $p_{A}$ because it is generated from $p_{A}$ by replacing a relatively small number of arcs with the same number of arcs of $p_{B}$. The advantage of EAX-1AB is its ability to better 
maintain the population diversity when used in an appropriate GA framework, which is presented in Algorithm 1 (see Section 2.2), resulting in a significant improvement in the solution quality. Another selection strategy (called EAX-Block) for selecting $A B$-cycles was also proposed by Nagata (2006b), but we do not use this variant of the EAX in this paper, because we could not find a significant improvement by using this strategy in our preliminary experiments.

\subsection{Main framework}

The main GA framework is shown in Algorithm 1. The search is initiated by generating $N_{\text {pop }}$ initial solutions (line 1). Here, we use a local search procedure using a variant of the 3-opt neighborhood to generate each of the initial population members. The detailed description of the local search procedure is presented in Section 2.3.

For each generation of the GA (lines 3-15), each population member is selected once, as both parent $p_{A}$ and parent $p_{B}$ in random order (lines 3 and 5 ). For each pair of parents, EAX-1AB generates offspring solutions, where $n_{c h}$ refers to the number of offspring solutions generated (line 6). Let $n_{c h}^{\max }$ be a parameter that specifies the maximum number of offspring solutions for each pair of parents. Therefore, $n_{c h}$ will be equal to the number of $A B$-cycles if the number of $A B$-cycles is less than $n_{c h}^{\max }$. Then, the best solution among the generated offspring solutions, denoted as $c_{\text {best }}$, is selected according to a given evaluation function (line 7). If the tour cost of $c_{\text {best }}$ is better than that of $p_{A}$ (line 8), it will replace one of the population members selected as $p_{A}$ or $p_{B}$ (line 10 and 12 ). If $c_{\text {best }}$ is more similar to $p_{A}$ than to $p_{B}$, the population member selected as $p_{A}$ is replaced (lines 9 and 10), where dist $(x, y)$ returns the number

of different arcs between the two tours. In contrast, if $c_{b e s t}$ is more similar to $p_{B}$ than to $p_{A}$ and the tour cost of $c_{\text {best }}$ is better than those of $p_{B}$ and $p_{A}$, the population member selected as $p_{B}$ is replaced (lines 11 and 12). Iterations of the generation are repeated until a termination condition is met (line 16). Finally, the best solution in the population is returned (line 17).

In the latest version of the GA using the EAX for the STSP by Nagata (2006b), the best offspring solution $\left(c_{\text {best }}\right)$ replaces only parent $p_{A}$ rather than both parents (i.e., lines 


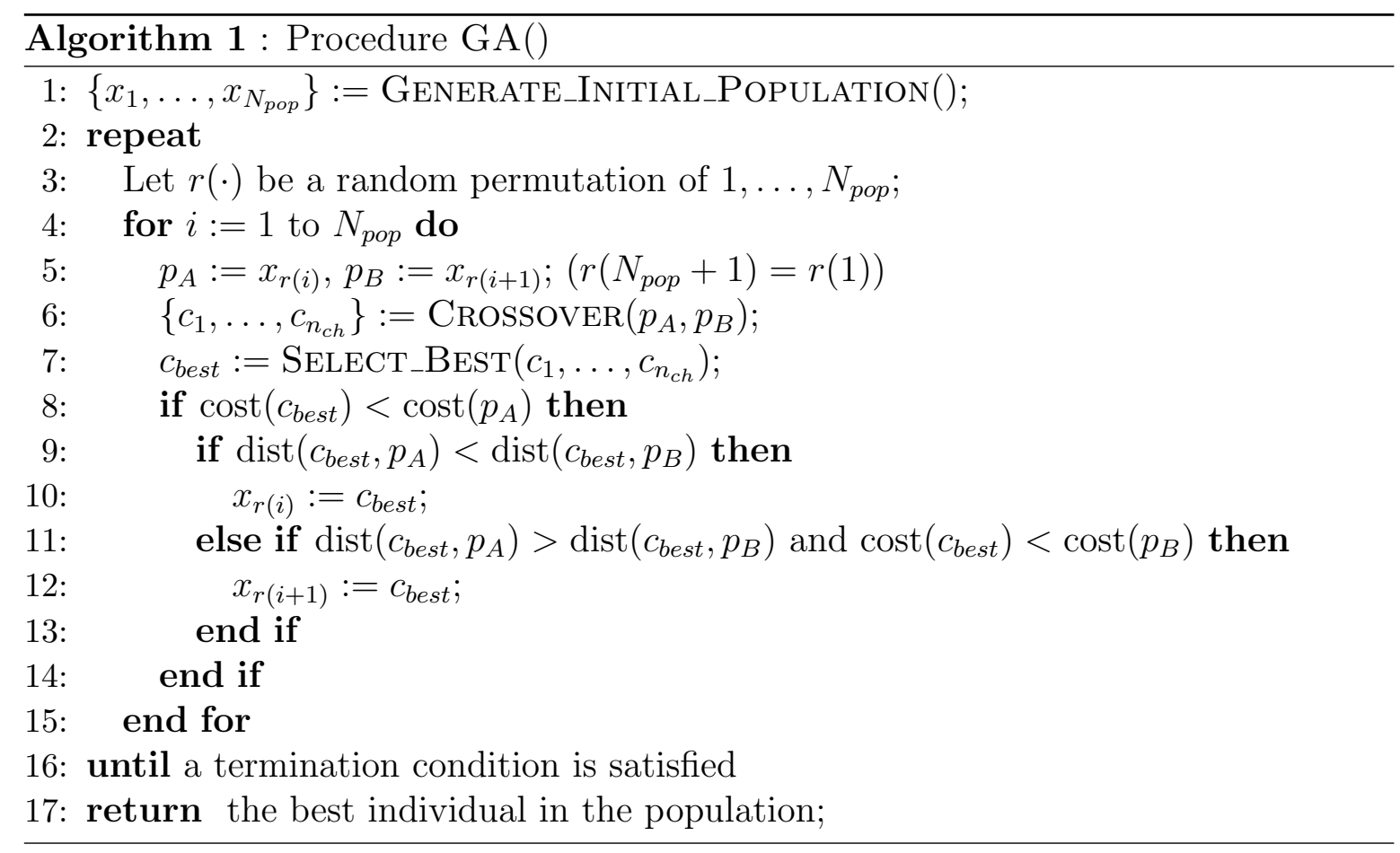

10, 11, and 13 are ignored). This selection strategy was introduced to better maintain the population diversity because EAX-1AB frequently generates offspring solutions that are more similar to $p_{A}$ than to $p_{B}$. We call this selection strategy "SEL1" in this paper. EAX-1AB adapted to the ATSP has the same property, although not to the same extent as EAX-1AB for the STSP. If $c_{\text {best }}$ is more similar to $p_{B}$ than to $p_{A}$ and $c_{b e s t}$ replaces $p_{A}$, the population diversity will be fairly reduced because two similar solutions remain in the population. Therefore, we add the additional selection mechanism that replaces $p_{B}$ rather than $p_{A}$ if $c_{\text {best }}$ is more similar to $p_{B}$ than to $p_{A}$. We call this selection strategy "SEL2" in this paper.

Although the most straightforward evaluation function for evaluating offspring solutions would be the tour cost, an alternative evaluation function was used in the latest version of the GA for the STSP to maintain the population diversity in a positive manner. The use of this evaluation function, however, did not improve the performance significantly in our preliminary experiments on ATSP instances. Therefore, we evaluate offspring solutions simply by the tour cost. 


\subsection{Local search}

The local search procedure used for generating the initial population is a simple hillclimbing method using a variant of the 3-opt neighborhood. For information on classical local search algorithms for the ATSP, we refer the reader to the work by Kanellakis and Papadimitriou (1980). The initial population consisting of $N_{\text {pop }}$ tours is generated by performing the local search procedure $N_{\text {pop }}$ times.

The 3-opt neighborhood used in this paper is defined as the tours that can be obtained from the current tour by replacing three arcs in other possible ways so that all arcs in the resulting tour have the same orientation. More precisely, let $\left(v_{1}, v_{2}\right)$, $\left(v_{3}, v_{4}\right)$, and $\left(v_{5}, v_{6}\right)$ denote three different arcs to be removed, and assume that $v_{3}$ appears after $v_{1}$ and before $v_{5}$ in the current tour. The three arcs to be added must be $\left(v_{1}, v_{4}\right),\left(v_{3}, v_{6}\right)$, and $\left(v_{5}, v_{2}\right)$ to make the arcs in the resulting tour have the same orientation. To speed up the local search procedure, we use a variant of the "don't look bits" strategy (Bentley, 1990). Let $\mathcal{N}(v)$ be a subset of the 3-opt neighborhood that requires $v=v_{1}$, and we call it a subneighborhood. To reduce the neighborhood size of a subneighborhood, $v_{4}$ is restricted to the vertices that are at most within the ten nearest from $v_{1}$. When using the don't look bits strategy, each of the vertices is assigned a flag having value true or false.

The local search procedure begins by generating a random tour and setting all flags to false. At each iteration, a tour that improves the current tour is searched in subneighborhoods whose associated flags are false. If an improving tour is found, the current tour is immediately moved to the new tour, and the flags of the three starting points of the added three edges are set to false. However, a flag of a vertex is set to true whenever no improving move is found in the corresponding subneighborhood. Iterations are repeated until all flags become true.

\section{Computational experiments}

In this section, we first perform the GA with several different configurations on the wellknown 27 instances included in TSPLIB to detect the best one. The results obtained 
with the best configuration are then compared for this set of 27 instances with those of the latest genetic algorithms proposed for the ATSP. Finally, we also run our GA on a set of 126 instances created in the work by Soler et al. (2008).

The GA was implemented in $\mathrm{C}++$ and was executed in a virtual machine environment (i.e., each job is executed on a single core, but multiple jobs may be executed in the same node) on a cluster with Intel Xeon 2.93-GHz nodes.

\subsection{Analysis of algorithm components}

We performed the proposed GA with four different configurations on the 27 instances in TSPLIB to detect the best configuration. Two parameters of Algorithm 1 are set as follows: $N_{\text {pop }}=100$ and $n_{c h}^{\max }=30$. Each run is terminated when the best solution is not improved over the last 20 generations. Four different configurations of the GA are summarized below.

SEL1+EAX-Rand Selection strategy SEL1 is used in Algorithm 1. EAX-Rand is used as the crossover operator. For each pair of parents, the number of offspring solutions is set to the number of $A B$-cycles if the number of $A B$-cycles generated is less than $n_{c h}^{\max }$. We use this restriction to compare EAX-Rand with EAX-1AB under the same condition.

SEL2+EAX-Rand Selection strategy SEL2 is used in Algorithm 1. EAX-Rand is used as the crossover operator. The number of offspring solutions is restricted as in the case of SEL1+EAX-Rand.

SEL1+EAX-1AB Selection strategy SEL1 is used in Algorithm 1. EAX-1AB is used as the crossover operator.

SEL2+EAX-1AB Selection strategy SEL2 is used in Algorithm 1. EAX-1AB is used as the crossover operator.

We performed the GA 100 times on each instance with each configuration. Table 1 shows results. The first column lists the instance names, where the number of vertices is indicated by the name except for kro124p (the number of vertices being 100 in 
this instance). For each of the GAs with four different configurations, we list the number of runs that succeeded in finding the optimal solution over 100 runs (Suc.), the average percentage excess with respect to the optimal solutions (a-err), and the average computation time per single run in seconds (a-T). Average results over all instances are also listed at the bottom of the table.

First, we compare the results of the GAs with two configurations, SEL1+EAXRand and SEL1+EAX-1AB. The table shows that the solution quality of the GA with EAX-1AB is better than that of the GA with EAX-Rand when selection strategy SEL1 is used. A major factor in this difference is that the population diversity is better maintained by replacing a population member (selected as $p_{A}$ ) with a relatively similar offspring solution and EAX-1AB usually generates offspring solutions similar to $p_{A}$. Next, we focus on the difference between selection strategies SEL1 and SEL2. The table shows that the use of selection strategy SEL2 improves the solution quality regardless of the crossover type. The main reason for this improvement is also that the population diversity is better maintained by replacing a population member with a relatively similar offspring solution and selection strategy SEL2 enhances this property. Here, one should note that SEL2+EAX-Rand is comparable to SEL2+EAX-1AB with respect to the solution quality, but SEL2+EAX-Rand requires more computation time than SEL2+EAX-1AB. This is because EAX-1AB can generate offspring solutions more efficiently than EAX-Rand, which is another advantage of EAX-1AB. By comparing the results listed in the table, we can see that SEL2-EAX-1AB seems to be a good configuration for the GA presented in Algorithm 1.

\subsection{Comparison with other algorithms}

We compare the results of our GA with configuration SEL2+EAX-1AB with those of the latest GAs or MAs proposed in the literature to solve the ATSP: the heuristics proposed by Choi et al. (2003), Buriol et al. (2004), and Xing et al. (2008). We make comparisons for the 27 well-known instances in TSPLIB. Table 2 lists the results; the algorithms are denoted as the reference on the first line of the table; this is followed by the type of method, the computer specifications, and the number of runs. For each 
Table 1: Results of the GAs with four different configurations.

\begin{tabular}{|c|c|c|c|c|c|c|c|c|c|c|c|c|}
\hline & \multicolumn{3}{|c|}{ SEL1+EAX-Rand } & \multicolumn{3}{|c|}{ SEL2+EAX-Rand } & \multicolumn{3}{|c|}{ SEL1+EAX-1AB } & \multicolumn{3}{|c|}{ SEL2+EAX-1AB } \\
\hline $\begin{array}{l}\text { Instance } \\
\end{array}$ & Suc. & a-err & $\mathrm{a}-\mathrm{T}$ & Suc. & a-err & $\mathrm{a}-\mathrm{T}$ & Suc. & a-err & $\mathrm{a}-\mathrm{T}$ & Suc. & a-err & $a-T$ \\
\hline br17 & 100 & 0.0000 & 0.00 & 100 & 0.0000 & 0.00 & 100 & 0.0000 & 0.00 & 100 & 0.0000 & 0.00 \\
\hline $\mathrm{p} 43$ & 92 & 0.0014 & 0.01 & 92 & 0.0014 & 0.04 & 91 & 0.0016 & 0.03 & 94 & 0.0011 & 0.02 \\
\hline ry $48 \mathrm{p}$ & 100 & 0.0000 & 0.01 & 100 & 0.0000 & 0.04 & 100 & 0.0000 & 0.02 & 100 & 0.0000 & 0.02 \\
\hline $\mathrm{ft} 53$ & 75 & 0.0275 & 0.02 & 93 & 0.0101 & 0.05 & 64 & 0.0408 & 0.02 & 95 & 0.0064 & 0.03 \\
\hline $\mathrm{ftv} 33$ & 100 & 0.0000 & 0.01 & 100 & 0.0000 & 0.02 & 100 & 0.0000 & 0.01 & 100 & 0.0000 & 0.01 \\
\hline $\mathrm{ftv} 35$ & 97 & 0.0041 & 0.01 & 100 & 0.0000 & 0.03 & 99 & 0.0014 & 0.01 & 100 & 0.0000 & 0.02 \\
\hline $\mathrm{ftv} 38$ & 92 & 0.0105 & 0.01 & 100 & 0.0000 & 0.03 & 99 & 0.0013 & 0.01 & 100 & 0.0000 & 0.02 \\
\hline $\mathrm{ftv} 44$ & 49 & 0.3162 & 0.02 & 62 & 0.2356 & 0.03 & 56 & 0.2728 & 0.02 & 66 & 0.2108 & 0.02 \\
\hline 47 & 100 & 0.0000 & 0.02 & 100 & 0.0000 & 0.03 & 100 & 0.0000 & 0.02 & 100 & 0.0000 & 0.02 \\
\hline ftv55 & 100 & 0.0000 & 0.02 & 100 & 0.0000 & 0.05 & 100 & 0.0000 & 0.03 & 100 & 0.0000 & 0.03 \\
\hline ftv64 & 100 & 0.0000 & 0.03 & 100 & 0.0000 & 0.05 & 100 & 0.0000 & 0.03 & 100 & 0.0000 & 0.04 \\
\hline $\mathrm{ft} 70$ & 67 & 0.0017 & 0.04 & 88 & 0.0006 & 0.08 & 69 & 0.0016 & 0.04 & 85 & 0.0008 & 0.04 \\
\hline ftv70 & 100 & 0.0000 & 0.03 & 100 & 0.0000 & 0.07 & 100 & 0.0000 & 0.04 & 100 & 0.0000 & 0.04 \\
\hline ftv90 & 75 & 0.0399 & 0.05 & 91 & 0.0114 & 0.10 & 85 & 0.0222 & 0.05 & 96 & 0.0057 & 0.05 \\
\hline & 79 & 0.0296 & 0.07 & 90 & 0.0112 & 0.11 & 87 & 0.0162 & 0.08 & 97 & 0.0034 & 0.06 \\
\hline kro12 & 93 & 0.0039 & 0.05 & 96 & 0.0012 & 0.16 & 96 & 0.0012 & 0.07 & 97 & 0.0009 & 0.08 \\
\hline ftv110 & 86 & 0.0179 & 0.09 & 87 & 0.0148 & 0.15 & 94 & 0.0072 & 0.10 & 93 & 0.0072 & 0.08 \\
\hline ftv120 & 79 & 0.0263 & 0.11 & 93 & 0.0069 & 0.22 & 92 & 0.0088 & 0.12 & 87 & 0.0157 & 0.10 \\
\hline ftv130 & 79 & 0.0243 & 0.08 & 87 & 0.0130 & 0.20 & 88 & 0.0130 & 0.13 & 89 & 0.0104 & 0.11 \\
\hline ftv140 & 75 & 0.0285 & 0.11 & 86 & 0.0124 & 0.18 & 84 & 0.0202 & 0.15 & 89 & 0.0116 & 0.12 \\
\hline 150 & 82 & 0.0146 & 0.11 & 88 & 0.0100 & 0.32 & 93 & 0.0065 & 0.18 & 91 & 0.0073 & 0.15 \\
\hline ftv160 & 92 & 0.0101 & 0.15 & 97 & 0.0041 & 0.26 & 96 & 0.0056 & 0.18 & 99 & 0.0011 & 0.17 \\
\hline $\mathrm{ftv} 170$ & 96 & 0.0044 & 0.16 & 96 & 0.0044 & 0.43 & 98 & 0.0022 & 0.24 & 98 & 0.0022 & 0.19 \\
\hline rbg323 & 100 & 0.0000 & 1.16 & 100 & 0.0000 & 3.59 & 100 & 0.0000 & 1.44 & 100 & 0.0000 & 0.96 \\
\hline rbg358 & 100 & 0.0000 & 1.65 & 100 & 0.0000 & 4.22 & 100 & 0.0000 & 1.82 & 100 & 0.0000 & 1.32 \\
\hline rbg 403 & 100 & 0.0000 & 1.82 & 100 & 0.0000 & 4.68 & 100 & 0.0000 & 1.86 & 100 & 0.0000 & 1.61 \\
\hline rbg 443 & 100 & 0.0000 & 1.76 & 100 & 0.0000 & 4.12 & 100 & 0.0000 & 1.77 & 100 & 0.0000 & 1.70 \\
\hline Average & 89.19 & 0.021 & 0.28 & 94.30 & 0.012 & 0.71 & 92.26 & 0.016 & 0.31 & 95.41 & 0.011 & 0.26 \\
\hline
\end{tabular}

algorithm, we list the number of runs that succeeded in finding the optimal solution (Suc.) if presented in the literature, the average percentage excess with respect to the optimal solutions (a-err), and the average computation time per single run in seconds (a-T). Results for the blank cells are not presented in the literature.

Table 2 shows that our GA is superior to all three compared algorithms in terms of average solution quality; only the procedure by Xing et al. seems to find similar solutions to ours (but they present results for only 16 out of the 27 instances), but their average results are slightly worse than ours. For the computation time, it is very difficult to make a fair comparison because the algorithms in the table were executed using different computers (and languages). Nonetheless, our GA would not be considerably slower than the compared heuristics even if the difference in computer speed is 
Table 2: Comparisons with other algorithms.

\begin{tabular}{|c|c|c|c|c|c|c|c|c|c|c|c|c|c|}
\hline Author & \multicolumn{2}{|c|}{$\begin{array}{c}\text { Choi at al. } \\
(2003)\end{array}$} & \multicolumn{3}{|c|}{$\begin{array}{c}\text { Buriol et al. } \\
(2004)\end{array}$} & \multicolumn{2}{|c|}{$\begin{array}{c}\text { Xing et al. } \\
(2008)\end{array}$} & \multicolumn{3}{|c|}{$\begin{array}{c}\text { Our method } \\
N_{\text {pop }}=100\end{array}$} & \multicolumn{3}{|c|}{$\begin{array}{c}\text { Our method } \\
N_{\text {pop }}=300\end{array}$} \\
\hline Method & \multicolumn{2}{|c|}{ GA } & \multicolumn{3}{|c|}{ MA } & \multicolumn{2}{|c|}{ MA } & \multicolumn{3}{|c|}{ GA } & \multicolumn{3}{|c|}{ GA } \\
\hline $\begin{array}{l}\text { Computer } \\
\text { \# of Runs }\end{array}$ & \multicolumn{2}{|c|}{$\begin{array}{c}\text { Pentium } \\
550 \mathrm{MHz} \\
3\end{array}$} & \multicolumn{3}{|c|}{$\begin{array}{c}\text { Pentium } \\
1.7 \mathrm{GHz} \\
20\end{array}$} & \multicolumn{2}{|c|}{$\begin{array}{c}\text { Pentium } \\
1.6 \mathrm{GHz} \\
100\end{array}$} & \multicolumn{3}{|c|}{$\begin{array}{c}\text { Xeon } \\
2.93 \mathrm{GHz} \\
100\end{array}$} & \multicolumn{3}{|c|}{$\begin{array}{c}\text { Xeon } \\
2.93 \mathrm{GHz} \\
100\end{array}$} \\
\hline Instance & a-err & $a-T$ & Suc. & a-err & $a-T$ & a-err & a- $T$ & Suc. & a-err & $\mathrm{a}-\mathrm{T}$ & Suc. & a-err & $\mathrm{a}-\mathrm{T}$ \\
\hline br17 & 0.00 & 0 & 20 & 0.00 & 0.05 & 0.000 & 1.9 & 100 & 0.000 & 0.00 & 100 & 0.0000 & 0.00 \\
\hline $\mathrm{ftv} 33$ & 0.00 & 4 & 20 & 0.00 & 0.05 & & & 100 & 0.000 & 0.01 & 100 & 0.0000 & 0.06 \\
\hline $\mathrm{ftv} 35$ & 0.14 & 1 & 20 & 0.00 & 0.08 & 0.000 & 15.6 & 100 & 0.000 & 0.02 & 100 & 0.0000 & 0.05 \\
\hline $\mathrm{ftv} 38$ & 0.13 & 2 & 5 & 0.10 & 0.26 & & & 100 & 0.000 & 0.02 & 100 & 0.0000 & 0.08 \\
\hline $\mathrm{p} 43$ & 0.00 & 1 & 11 & 0.01 & 0.35 & 0.000 & 4.2 & 94 & 0.001 & 0.02 & 100 & 0.0000 & 0.10 \\
\hline $\mathrm{ftv} 44$ & 1.30 & 5 & 7 & 0.44 & 0.36 & & & 66 & 0.211 & 0.02 & 94 & 0.0372 & 0.07 \\
\hline $\mathrm{ftv} 47$ & 0.00 & 3 & 20 & 0.00 & 0.13 & 0.000 & 90.3 & 100 & 0.000 & 0.02 & 100 & 0.0000 & 0.07 \\
\hline ry $48 p$ & 0.33 & 5 & 17 & 0.03 & 0.32 & 0.001 & 53.4 & 100 & 0.000 & 0.02 & 100 & 0.0000 & 0.07 \\
\hline $\mathrm{ft} 53$ & 0.00 & 8 & 20 & 0.00 & 0.2 & 0.000 & 41.2 & 95 & 0.006 & 0.03 & 100 & 0.0000 & 0.09 \\
\hline $\mathrm{ftv} 55$ & 0.00 & 5 & 20 & 0.00 & 0.16 & 0.000 & 125.6 & 100 & 0.000 & 0.03 & 100 & 0.0000 & 0.10 \\
\hline $\mathrm{ftv} 64$ & 0.00 & 7 & 20 & 0.00 & 0.24 & 0.000 & 101.2 & 100 & 0.000 & 0.04 & 100 & 0.0000 & 0.17 \\
\hline $\mathrm{ft} 70$ & 0.21 & 20 & 8 & 0.03 & 0.86 & 0.000 & 83.6 & 85 & 0.001 & 0.04 & 100 & 0.0000 & 0.14 \\
\hline $\mathrm{ftv} 70$ & 0.00 & 12 & 19 & 0.01 & 0.38 & 0.000 & 43.8 & 100 & 0.000 & 0.04 & 100 & 0.0000 & 0.19 \\
\hline ftv90 & 0.00 & 20 & 20 & 0.00 & 0.28 & & & 96 & 0.006 & 0.05 & 100 & 0.0000 & 0.15 \\
\hline ftv100 & 0.00 & 62 & 20 & 0.00 & 0.40 & & & 97 & 0.003 & 0.06 & 100 & 0.0000 & 0.29 \\
\hline kro124p & 0.52 & 67 & 18 & 0.01 & 0.74 & 0.001 & 28.9 & 97 & 0.001 & 0.08 & 100 & 0.0000 & 0.33 \\
\hline ftv110 & 0.54 & 61 & 18 & 0.02 & 0.98 & & & 93 & 0.007 & 0.08 & 100 & 0.0000 & 0.36 \\
\hline $\mathrm{ftv} 120$ & 0.29 & 89 & 7 & 0.14 & 2.00 & & & 87 & 0.016 & 0.10 & 100 & 0.0000 & 0.37 \\
\hline ftv130 & 0.59 & 97 & 18 & 0.01 & 1.30 & & & 89 & 0.010 & 0.11 & 100 & 0.0000 & 0.42 \\
\hline ftv140 & 0.32 & 81 & 14 & 0.08 & 2.11 & & & 89 & 0.012 & 0.12 & 100 & 0.0000 & 0.53 \\
\hline $\mathrm{ftv} 150$ & 0.55 & 88 & 18 & 0.01 & 1.54 & & & 91 & 0.007 & 0.15 & 100 & 0.0000 & 0.56 \\
\hline ftv160 & 0.12 & 101 & 16 & 0.02 & 2.04 & & & 99 & 0.001 & 0.17 & 100 & 0.0000 & 0.65 \\
\hline ftv170 & 0.22 & 94 & 15 & 0.05 & 2.78 & 0.020 & 68.3 & 98 & 0.002 & 0.19 & 100 & 0.0000 & 0.69 \\
\hline rbg323 & 0.00 & 2 & 20 & 0.00 & 0.07 & 0.000 & 110.4 & 100 & 0.000 & 0.96 & 100 & 0.0000 & 4.25 \\
\hline rbg358 & 0.00 & 3 & 20 & 0.00 & 0.08 & 0.000 & 58.4 & 100 & 0.000 & 1.32 & 100 & 0.0000 & 5.63 \\
\hline rbg403 & 0.00 & 2 & 20 & 0.00 & 0.08 & 0.000 & 33.1 & 100 & 0.000 & 1.61 & 100 & 0.0000 & 6.63 \\
\hline $\operatorname{rbg} 443$ & 0.00 & 4 & 20 & 0.00 & 0.09 & 0.000 & 144.2 & 100 & 0.000 & 1.70 & 100 & 0.0000 & 6.74 \\
\hline
\end{tabular}

considered, and it seems that the MA by Xing et al. would require longer computation time. We additionally performed our GA with a population size of 300 to improve the solution quality. Our GA with a population size of 300 found optimal solutions in all 100 runs for 26 out of the 27 TSPLIB instances (94 on ftv44). The computation time was increased by about a factor of 3 , so our GA with a population size of 300 would still be faster than the MA by Xing et al., but in this case with a much greater difference in our favor for the average deviations. 


\subsection{Other results}

As mentioned in Section 1, many single-vehicle routing problems that actually cannot be modeled as an ATSP can be solved through a polynomial transformation into an ATSP. This is the case of the problem presented by Soler et al. (2008). They generated 128 instances for that problem and they transformed them into ATSP instances. To solve the transformed instances, they run the ATSP exact procedure by Corberan et al. (2005) on a PC with 1.8-GHz Pentium IV processor. Most of the instances were optimally solved in less than $2 \mathrm{~h}$ of running time, some instances needed more than $10 \mathrm{~h}$ to obtain the optimal solution (with one of them taking more than $17 \mathrm{~h}$ ), and in only two instances was the execution aborted owing to the long period of time spent without finding the optimal cost.

Thus, we have a set of 126 ATSP instances with known optimal solution obtained from the aforementioned work, and with a number of vertices between 64 and 315. We have uploaded all data corresponding to this set of instances to the website http://www.iumpa.upv.es/arxivDSoler/, to make them easily available to any researcher.

This set has been recently used by Bräysy et al. (2011) as a benchmark set to test the efficiency of a memetic algorithm for a capacitated vehicle routing problem. Although this algorithm was not specifically designed to solve problems with a single vehicle, it was able to find the optimal solution in 86 out of the 126 instances.

In Table 3, we show the results on this set of ATSP instances obtained with our GA with configuration SEL2+EAX-1AB where the population size was set to 300. In this table, the 126 instances are partitioned into 24 groups, separated by horizontal lines, according to the features of the original instances from which they come. For each instance the table lists the name of the original instance (Name); the number of vertices $(|V|)$; the optimal cost (Opt-cost); the time in seconds to obtain the optimal solution with the exact procedure (Opt-T); the best solution found in 100 runs of the GA (Best), with Opt shown if this solution coincides with the optimal one; the average cost over the 100 runs (a-cost), with Opt meaning that the GA has reached the optimal solution in the 100 runs; and finally the average computation time per single run in 
seconds (a-T).

From Table 3 we can see that our GA was able to find the optimal solution in 121 out of the 126 instances. The worst case was instance D143042, where the percentage excess of the best solution obtained with our GA over the optimal cost is only $0.0021 \%$, and the second worst case was D183841b, where the percentage excess of the best solution obtained with our GA over the optimal cost is only $0.0004 \%$. Moreover, with respect to the instances where the GA did not find the optimal solution in all 100 runs, the worst average deviation of the 100 runs with respect to the optimal cost also corresponds to instance D143042 (0.0024\%).

Table 3: Computational results for instances from Soler et al. (2008).

\begin{tabular}{lrrrrrr}
\hline Name & $|V|$ & Opt-cost & Opt-T & Best & a-cost & a-T \\
\hline D41140 & 64 & 417880 & 6.31 & Opt & Opt & 0.2 \\
D61440 & 77 & 512674 & 14.22 & Opt & Opt & 0.3 \\
D4940m & 51 & 336325 & 2.69 & Opt & Opt & 0.1 \\
D81740m & 102 & 664613 & 5.65 & Opt & Opt & 0.4 \\
D4940 & 67 & 422508 & 6.26 & Opt & Opt & 0.2 \\
D81740 & 129 & 809706 & 51.74 & Opt & Opt & 0.6 \\
\hline D82040m & 103 & 689971 & 12.19 & Opt & Opt & 0.5 \\
D102240m & 121 & 803595 & 34.54 & Opt & Opt & 0.6 \\
D82040 & 122 & 790971 & 8.57 & Opt & Opt & 0.7 \\
D102240 & 163 & 1027751 & 204.87 & Opt & Opt & 1.0 \\
\hline D102640m & 118 & 815096 & 151.26 & Opt & Opt & 1.3 \\
D122640m & 130 & 880335 & 21.7 & Opt & Opt & 1.3 \\
D122940m & 148 & 997772 & 765.55 & Opt & Opt & 1.1 \\
D102640 & 139 & 926142 & 330.59 & Opt & Opt & 1.8 \\
D122640 & 150 & 990448 & 69.15 & Opt & Opt & 1.1 \\
D122940 & 209 & 1316922 & 1018.59 & Opt & 1316922.2 & 2.6 \\
\hline D143040a & 161 & 1072673 & 105.4 & Opt & Opt & 1.5 \\
D143340 & 230 & 1452339 & 767.64 & Opt & Opt & 4.7 \\
D143340m & 162 & 1100177 & 585.72 & Opt & Opt & 1.4 \\
D143040 & 236 & 1472328 & 97.22 & Opt & 1472332.6 & 3.3 \\
D143040b & 186 & 1210175 & 185.7 & Opt & Opt & 3.2 \\
\hline D163440a & 169 & 1145914 & 181.96 & Opt & Opt & 1.4 \\
D163440 & 226 & 1452914 & 1519.4 & Opt & Opt & 0.9 \\
D163440b & 200 & 1310767 & 197.4 & Opt & 1310767.3 & 2.4 \\
D183840a & 184 & 1255781 & 305.49 & Opt & Opt & 2.9 \\
D163740 & 226 & 1468709 & 2585.73 & Opt & Opt & 4.3 \\
D183840 & 258 & 1652091 & 1171.17 & Opt & Opt & 5.9 \\
D183840b & 220 & 1449912 & 565.84 & Opt & Opt & 4.3 \\
\hline D204240a & 213 & 1438448 & 108.65 & Opt & 1438453.5 & 2.5 \\
D184040a & 196 & 1331558 & 3467.28 & Opt & Opt & 2.4 \\
D204240 & 315 & 1980807 & 408.64 & Opt & 1980814.5 & 8.3 \\
D184040 & 262 & 1686092 & 3119.43 & Opt & Opt & 6.7 \\
D184040b & 226 & 1493759 & 1037.65 & Opt & 1493776.2 & 4.5 \\
D204240b & 275 & 1764636 & 1126.68 & Opt & Opt & 5.1 \\
\hline D41141 & 68 & 454381 & 28.73 & Opt & Opt & 0.3
\end{tabular}


Table 3: Computational results for instances from Soler et al. (2008).

\begin{tabular}{|c|c|c|c|c|c|c|}
\hline Name & $|V|$ & Opt-cost & Opt-T & Best & a-cost & $\mathrm{a}-\mathrm{T}$ \\
\hline D4941 & 70 & 452101 & 12.97 & Opt & 452107.4 & 0.3 \\
\hline D61441 & 84 & 561657 & 17.52 & Opt & $\mathrm{Opt}$ & 0.4 \\
\hline D61641 & 95 & 633760 & 72.12 & Opt & Opt & 0.5 \\
\hline D81741 & 112 & 730893 & 67.99 & Opt & 730893.9 & 0.8 \\
\hline D82041m & 122 & 804695 & 999.92 & Opt & Opt & 0.7 \\
\hline D102241m & 128 & 854290 & 151.98 & Opt & Opt & 0.8 \\
\hline D82041 & 136 & 880751 & 104.35 & Opt & Opt & 0.9 \\
\hline D102241 & 161 & 1029382 & 658.94 & Opt & $\mathrm{Opt}$ & 1.9 \\
\hline D122641a & 152 & 1011650 & 444.4 & Opt & $\mathrm{Opt}$ & 1.4 \\
\hline D102641m & 152 & 1009032 & 633.89 & Opt & Opt & 1.2 \\
\hline D122941m & 157 & 1058436 & 212.95 & Opt & Opt & 1.3 \\
\hline D122641 & 207 & 1306865 & 378.99 & Opt & Opt & 3.1 \\
\hline D102641 & 188 & 1199118 & 1006.29 & Opt & 1199121.2 & 2.3 \\
\hline D122941 & 192 & 1243493 & 388.99 & Opt & Opt & 1.9 \\
\hline D122641b & 179 & 1156769 & 367.12 & Opt & Opt & 2.0 \\
\hline D143041a & 164 & 1103917 & 166.81 & Opt & 1103917.2 & 2.5 \\
\hline D143341a & 178 & 1201011 & 40.43 & Opt & 1201016.0 & 2.3 \\
\hline D143341b & 211 & 1376011 & 3313.77 & Opt & 1376020.6 & 2.6 \\
\hline D143041 & 217 & 1389294 & 81.79 & 1389296 & 1389296.0 & 4.3 \\
\hline D143341 & 251 & 1584011 & 2085.68 & Opt & 1584022.4 & 5.0 \\
\hline D143041b & 185 & 1219063 & 213.49 & Opt & Opt & 3.1 \\
\hline D163441a & 198 & 1313082 & 853.43 & Opt & Opt & 2.8 \\
\hline D163441 & 282 & 1761528 & 886.66 & Opt & 1761554.6 & 8.7 \\
\hline D163441b & 242 & 1547424 & 1253.51 & Opt & Opt & 2.8 \\
\hline $\mathrm{D} 163741 \mathrm{~m}$ & 200 & 1343095 & 2447.26 & Opt & Opt & 2.6 \\
\hline D163741 & 235 & 1530323 & 928.57 & Opt & 1530329.7 & 7.1 \\
\hline D183841a & 206 & 1385166 & 1724.55 & Opt & $\mathrm{Opt}$ & 3.8 \\
\hline D183841 & 278 & 1769642 & 970.48 & 1769648 & 1769648.0 & 7.5 \\
\hline D183841b & 237 & 1552379 & 977.39 & 1552385 & 1552385.0 & 5.1 \\
\hline D184041b & 242 & 1591521 & 1054.9 & Opt & Opt & 5.3 \\
\hline D184041a & 224 & 1491653 & 1180.46 & Opt & Opt & 5.0 \\
\hline D184041 & 282 & 1803657 & 315.32 & Opt & $\mathrm{Opt}$ & 7.4 \\
\hline D204241a & 224 & 1510820 & 503.23 & Opt & 1510820.9 & 4.1 \\
\hline D204441a & 230 & 1556414 & 460.77 & Opt & Opt & 4.1 \\
\hline D204241 & 291 & 1872362 & 5613.99 & Opt & 1872363.1 & 6.4 \\
\hline D204441 & 315 & 2007744 & 5536.11 & Opt & 2007778.5 & 9.2 \\
\hline D204241b & 258 & 1693166 & 6030.21 & Opt & 1693169.9 & 5.4 \\
\hline D204441b & 273 & 1785585 & 1125.2 & Opt & 1785588.3 & 7.3 \\
\hline D4942 & 80 & 517768 & 12.91 & Opt & Opt & 0.4 \\
\hline D61642 & 138 & 875072 & 98.32 & Opt & Opt & 1.1 \\
\hline D61442 & 120 & 768117 & 145.17 & Opt & Opt & 0.7 \\
\hline D81742 & 127 & 828368 & 252.99 & Opt & Opt & 1.0 \\
\hline D82042 & 128 & 849394 & 190.2 & Opt & Opt & 0.9 \\
\hline D102242 & 146 & 963636 & 1941.23 & Opt & 963636.6 & 1.5 \\
\hline D122642m & 162 & 1077557 & 945.37 & Opt & 1077562.4 & 1.6 \\
\hline $\mathrm{D} 122942 \mathrm{~m}$ & 172 & 1149670 & 1966.17 & Opt & 1149674.2 & 2.2 \\
\hline D102642 & 160 & 1065324 & 275.34 & Opt & 1065326.2 & 1.9 \\
\hline D122642 & 176 & 1153625 & 3036.56 & Opt & 1153629.6 & 2.2 \\
\hline D122942 & 188 & 1235878 & 623.02 & Opt & 1235882.4 & 4.2 \\
\hline D143042m & 184 & 1224644 & 942.36 & Opt & 1224664.1 & 3.6 \\
\hline $\mathrm{D} 143342 \mathrm{~m}$ & 200 & 1329993 & 184.33 & Opt & 1329995.5 & 3.4 \\
\hline
\end{tabular}


Table 3: Computational results for instances from Soler et al. (2008).

\begin{tabular}{|c|c|c|c|c|c|c|}
\hline Name & $|V|$ & Opt-cost & Opt-T & Best & a-cost & $\mathrm{a}-\mathrm{T}$ \\
\hline D143042 & 211 & 1369730 & 298.3 & 1369759 & 1369763.5 & 4.8 \\
\hline D143342 & 237 & 1525120 & 641.75 & 1525122 & 1525122.0 & 4.4 \\
\hline D163442 & 253 & 1622974 & 593.09 & Opt & 1622984.5 & 5.3 \\
\hline D163442a & 206 & 1367607 & 512.45 & Opt & Opt & 3.1 \\
\hline D163742a & 214 & 1432892 & 8394.27 & Opt & 1432921.6 & 6.6 \\
\hline D163442b & 219 & 1442855 & 4401.89 & Opt & 1442857.0 & 3.6 \\
\hline D163742b & 246 & 1602916 & 3442.84 & Opt & 1602952.5 & 4.7 \\
\hline D163742 & 301 & 1888149 & 5267.52 & Opt & 1888173.4 & 8.8 \\
\hline D183842m & 213 & 1434387 & 586.55 & Opt & 1434407.1 & 4.9 \\
\hline D183842 & 233 & 1542727 & 251.83 & Opt & 1542740.9 & 7.2 \\
\hline D184042m & 229 & 1534827 & 6919.67 & Opt & Opt & 3.8 \\
\hline D184042 & 270 & 1753907 & 6398.55 & Opt & Opt & 5.8 \\
\hline D204242a & 236 & 1587087 & 1284.93 & Opt & 1587089.3 & 4.6 \\
\hline D204242 & 289 & 1872624 & 5832.32 & Opt & 1872626.1 & 10.8 \\
\hline D204242b & 265 & 1742305 & 3821.49 & Opt & 1742312.0 & 6.9 \\
\hline D41143 & 108 & 695196 & 402.88 & Opt & Opt & 0.7 \\
\hline D61443 & 128 & 822217 & 240.52 & Opt & 822217.9 & 1.1 \\
\hline D61643 & 134 & 869353 & 2003.02 & Opt & Opt & 1.5 \\
\hline D81743 & 147 & 948708 & 741.11 & Opt & 948719.1 & 2.1 \\
\hline D82043m & 154 & 1003444 & 1497.6 & Opt & 1003444.2 & 1.6 \\
\hline D82043 & 168 & 1079720 & 133.91 & Opt & Opt & 2.1 \\
\hline D102643 & 168 & 1121130 & 2091.13 & Opt & Opt & 2.2 \\
\hline D122643 & 186 & 1218825 & 131.72 & Opt & Opt & 2.2 \\
\hline D122943m & 210 & 1365993 & 554.48 & Opt & Opt & 1.2 \\
\hline D122943 & 240 & 1526097 & 2901.27 & Opt & Opt & 7.1 \\
\hline D4944 & 120 & 758785 & 5141.3 & Opt & 758791.0 & 1.0 \\
\hline D61444 & 152 & 964730 & 2109.14 & Opt & 964740.4 & 1.8 \\
\hline D61644 & 153 & 984935 & 1924.15 & Opt & 984937.0 & 1.9 \\
\hline D81744 & 154 & 995860 & 13342.23 & Opt & 995865.8 & 2.2 \\
\hline D102244m & 194 & 1245433 & 1997.75 & Opt & 1245436.6 & 3.7 \\
\hline D82044 & 168 & 1091866 & 1016.54 & Opt & Opt & 2.5 \\
\hline D122644m & 198 & 1295659 & 63854.52 & Opt & 1295663.4 & 3.8 \\
\hline D122944m & 210 & 1380447 & 6368.56 & Opt & 1380447.9 & 6.4 \\
\hline D122944 & 224 & 1456564 & 14789.84 & Opt & 1456573.8 & 8.4 \\
\hline D102644 & 214 & 1379555 & 35721.07 & Opt & 1379562.2 & 3.8 \\
\hline D143044m & 219 & 1438672 & 225.13 & Opt & 1438704.1 & 9.9 \\
\hline D143044 & 245 & 1578725 & 4017.8 & Opt & 1578759.7 & 9.4 \\
\hline D143344 & 236 & 1549308 & 39896.66 & Opt & Opt & 6.4 \\
\hline D163744m & 254 & 1671485 & 37142.93 & Opt & Opt & 13.8 \\
\hline D163744 & 278 & 1801619 & 50808.44 & Opt & 1801621.8 & 16.7 \\
\hline
\end{tabular}

\section{Conclusions}

We have presented a new GA to solve the ATSP. We have checked its efficiency on a set of 153 benchmark ATSP instances with known optimal solution. From the obtained results with the GA, we believe that our procedure is very competitive. In fact, it outper- 
forms the results obtained with previous ATSP heuristics in the well-known set of ATSP instances from TSPLIB. Because the ATSP can be used to solve many real-world problems, either directly or through a transformation, our aim is to show that the procedure presented here can be useful for solving these problems or for measuring the efficiency of actual or future proposed heuristics that directly address these problems. Moreover, to strengthen this aim, through the website http://www.iumpa.upv.es/arxivDSoler/ we make easily available to any researcher, a detailed information on the set of 126 ATSP instances used here and in previous papers and not coming from TSPLIB.

\section{Acknowledgements}

This work has been partially supported by the Ministerio de Educación y Ciencia of Spain (Project No. TIN2008-06441-C02-01).

\section{References}

Albayrak, M., \& Allahverdi, N. (2011). Development a new mutation operator to solve the traveling salesman problem by aid of genetic algorithms. Expert Systems with Applications, 38, 1313-1320.

Albiach, J., Sanchis, J.M., \& Soler, D. (2008). An asymmetric TSP with time windows and with time-dependent travel times and costs: An exact solution through a graph transformation. European Journal of Operational Research, 189, 789-802.

Baldacci, R., Bartolini, E., \& Laporte, G. (2010). Some applications of the generalized vehicle routing problem. Journal of the Operational Research Society, 61, 10721077 .

Ben-Arieh, D., Gutin, G., Penn, M., Yeo, A., \& Zverovitch, A. (2003). Transformations of generalized ATSP into ATSP. Operations Research Letters, 31, 357-365.

Bentley, J.L. (1990). Experiments on traveling salesman heuristics, Proceedings of the First Annual ACM-SIAM Symposium on Discrete Algorithms, pp. 91-99.

Blais, M., \& Laporte, G. (2003). Exact solution of the generalized routing problem through graph transformations. Journal of the Operational Research Society, 54, 906-910.

Bräysy, O., Martínez, E., Nagata, Y., \& Soler, D. (2011). The mixed capacitated general routing problem with turn penalties. Expert Systems with Applications, 30, 12954-12966. 
Buriol, L., França, P.M., \& Moscato, P. (2004). A new memetic algorithm for the asymmetric traveling salesman problem. Journal of Heuristics, 10, 483-506.

Chen, S.M., \& Chien, C.Y. (2011). Parallelized genetic ant colony systems for solving the traveling salesman problem. Expert Systems with Application, 38, 3873-3883.

Choi, I.C., Kim, S.I., \& Kim, H.S. (2003). A genetic algorithm with a mixed region search for the asymmetric traveling salesman problem. Computers $\& 5$ Operations Research, 30, 773-786.

Clossey, J., Laporte, G., \& Soriano, P. (2001). Solving arc routing problems with turn penalties. Journal of the Operational Research Society, 52, 433-439.

Corberán, A., Martí, R., Martínez, E., \& Soler, D. (2002). The rural postman problem on mixed graphs with turn penalties. Computers \& Operations Research, 29, 887-903.

Corberán, A., Mejía, G., \& Sanchis, J.M. (2005). New results on the mixed general routing problem. Operations Research, 53, 363-376.

Fischetti, M., Lodi, A., \& Toth, P. (2002). Exact methods for the asymmetric traveling salesman problem. In: G. Gutin \& A.P. Punnen (Eds.), The Traveling Salesman Problem and Its Variations, pp. 168-205 (Boston: Kluwer).

Kanellakis, P.C., \& Papadimitriou, C.H. (1980). Local search for the asymmetric traveling salesman problem. Operations Research, 28, 1086-1099.

Laporte, G. (1997). Modeling and solving several classes of arc routing problems as traveling salesman problems. Computers \& Operations Research, 24, 1057-1061.

Laporte, G. (2010). A concise guide to the traveling salesman problem. Journal of the Operational Research Society, 61, 35-40.

Micó, J.C., \& Soler, D. (2011). The capacitated general windy routing problem with turn penalties. Operations Research Letters, 39, 265-271.

Nagata, Y., \& Kobayashi, S. (1997). Edge assembly crossover: A high-power genetic algorithm for the traveling salesman problem. Proceedings of the 7th International Conference on Genetic Algorithms, pp. 450-457.

Nagata, Y. (2004). The EAX algorithm considering diversity loss. Proceedings of the 8th International Conference on Parallel Problem Solving from Nature, Lecture Notes in Computer Science, 3242, 332-341.

Nagata, Y. (2006a). Fast EAX algorithm considering population diversity for traveling salesman problems. Proceedings of the 6th International Conference on Evolutionary Computation in Combinatorial Optimization, Lecture Notes in Computer Science, 3906, 171-182.

Nagata, Y. (2006b). New EAX crossover for large TSP instances. Proceedings of the 9th International Conference on Parallel Problem Solving from Nature, Lecture Notes in Computer Science, 4193, 372-381. 
Noon, C.E., \& Bean, J.C. (1993). An efficient transformation of the generalized traveling salesman problem. INFOR, 31, 39-44.

Oncan, T., Altinel, I.K., \& Laporte, G. (2009). A comparative analysis of several asymmetric traveling salesman problem formulations. Computers $\&$ Operations Research, 36, 637-654.

Rego, C., Gamboa, D., Glover, F., \& Osterman, C. (2011). Traveling salesman problem heuristics: Leadings methods, implementations and latest advances. European Journal of Operational Research, 211, 427-441.

Soler, D., Albiach, J., \& Martínez, E. (2009). A way to optimally solve a timedependent vehicle routing problem with time windows. Operations Research Letters, 37, 37-42.

Soler, D., Martínez, E., \& Micó, J.C. (2008). A transformation for the mixed general routing problem with turn penalties. Journal of the Operational Research Society, $59,540-547$.

Xing, L.N., Chen, Y.W., Yang, K.W., How, F., Shen, X.S., \& Cai, H.P. (2008). A hybrid approach combining an improved genetic algorithm and optimization strategies for the asymmetric traveling salesman problem. Engineering Applications of Artificial Intelligence, 21, 1370-1380. 

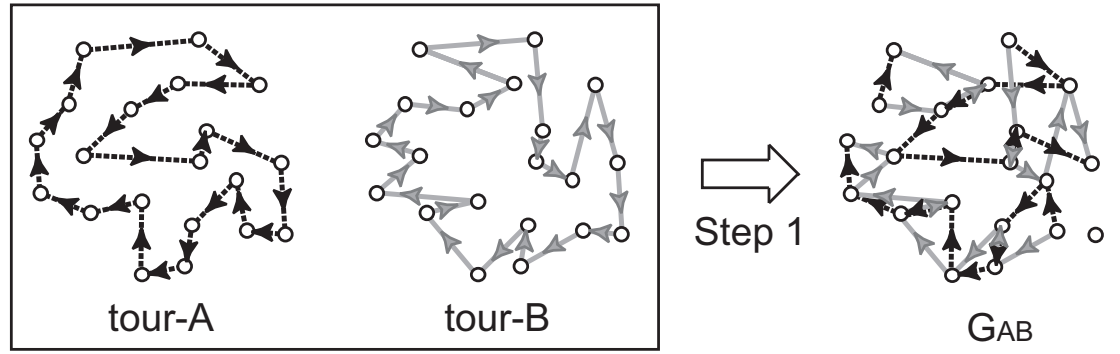

$\square$ Step 2

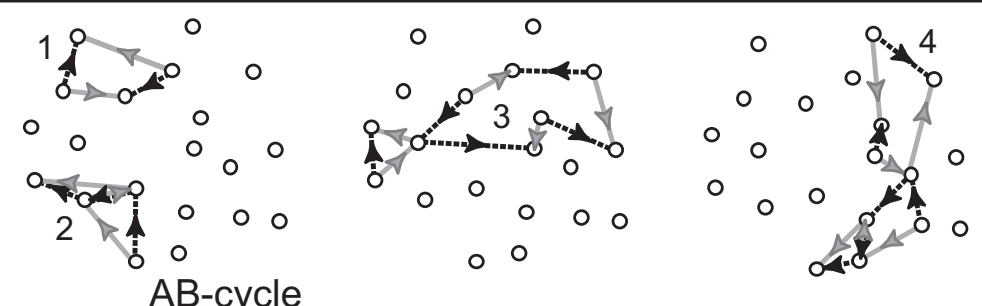

AB-cycle

$\lceil$ Step 3

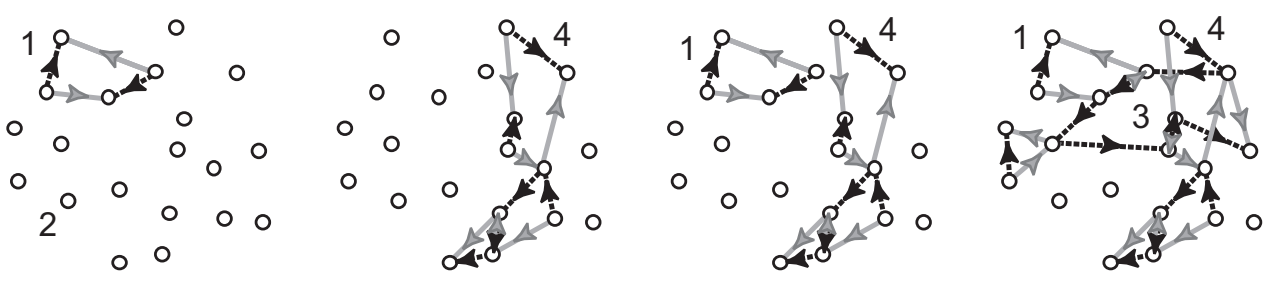

\section{E-set}

$\lceil$ Step 4
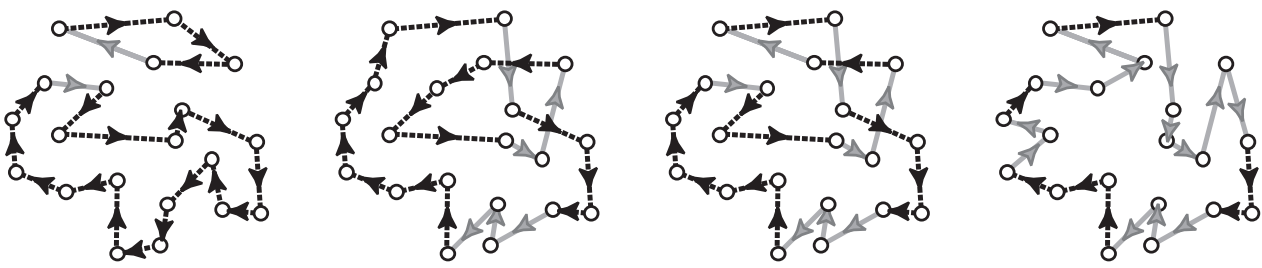

Intermediate
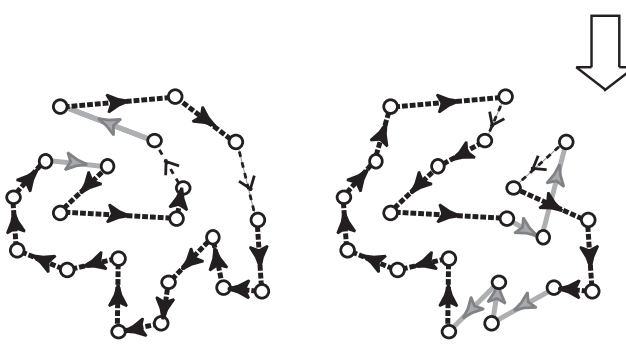

Step 5
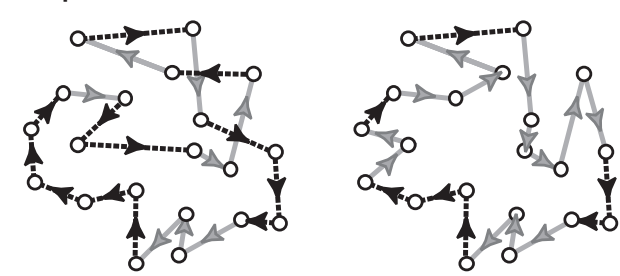

valid tour

Figure 1: Illustration of the EAX for the ATSP. 